\title{
Mesotelioma de túnica vaginal en paciente con hidrocele rápidamente progresivo
}

\section{Mesothelioma of the Tunica Vaginalis in a Patient with Rapidly Progressive Hydrocele}

\author{
Adolfo Serrano $^{1}$ Alejandra Bravo-Balado ${ }^{1}$ Diana Cañón ${ }^{2}$ Daniela Robledo ${ }^{1}$ Liliana Fonseca ${ }^{3}$ \\ ${ }^{1}$ Departmento de Urología, Hospital Universitario Fundación Santa Fe \\ de Bogotá y Facultad de Medicina, Universidad de los Andes, Bogotá \\ D.C., Colombia \\ 2 Departmento de Patología, Hospital Universitario Fundación Santa \\ Fe de Bogotá y Facultad de Medicina, Universidad de los Andes, \\ Bogotá D.C., Colombia \\ Address for correspondence Adolfo Serrano, MD, Departamento de \\ Urología, Hospital Universitario Fundación Santa Fe de Bogotá y \\ Facultad de Medicina, Universidad de los Andes, Carrera 7 No. 118-09, \\ Unidad Renal, piso 3, 110111, Bogotá D.C., Colombia \\ (e-mail: adolfo.serrano@ama.com.co; \\ investigacion.urologia@fsfb.org.co).
}

${ }^{3}$ Departmento de Urología, Hospital Universitario Fundación Santa Fe de Bogotá, Bogotá D.C., Colombia

Urol Colomb 2019;28:66-69.

\section{Resumen \\ Palabras clave \\ - hidrocele \\ - linfadenectomía retroperitoneal \\ - mesotelioma \\ - testículo \\ - túnica vaginal}

El mesotelioma es una entidad poco común y agresiva que compromete el mesotelio, involucrando así los diferentes órganos cubiertos por membranas serosas. El mesotelioma de túnica vaginal es una neoplasia muy rara, con un cuadro clínico poco específico y de difícil diagnóstico con las técnicas imagenológicas actuales. Presentamos el caso de un paciente con hidrocele rápidamente progresivo que fue llevado a hidrocelectomía. Se realizó un diagnóstico histopatológico de mesotelioma epitelial maligno de túnica vaginal que requirió orquidectomía radical, hemiescrotomectomía y linfadenectomía retroperitoneal. A un año de seguimiento, el paciente no tiene evidencia de la enfermedad.

Mesothelioma is an uncommon and aggressive entity that compromises the mesothelium, thus involving the different organs covered by serous membranes. The mesothelioma of the tunica vaginalis is a very rare neoplasm, with a non-specific clinical picture and difficult diagnosis with current imaging techniques. We report the case of a patient with rapidly progressive hydrocele who was taken to hydrocelectomy. A histopathological diagnosis of epithelial mesothelioma of the tunica vaginalis was made, which required radical orchiectomy, hemiscrotectomy and retroperitoneal lymphadenectomy. At one year of follow-up, the patient has no evidence of the disease.

\section{Introducción}

El mesotelioma es un tumor raro derivado de las células mesoteliales. La afectación testicular se deriva de la túnica vaginal que desciende con los testículos hacia el escroto durante el desarrollo fetal. ${ }^{1}$ El mesotelioma de túnica vaginal comprende menos del $1 \%$ de todos los mesoteliomas, lo cual explica que haya pocos reportes en la literatura. Puede ocurrir a cualquier edad, pero hay un pico de incidencia entre los $55 \mathrm{y}$ los 75 años. ${ }^{2}$ Más de la mitad de los pacientes presenta hidrocele o una masa escrotal y en hasta el $66 \%$ de ellos hay antecedente de exposición a asbestos. ${ }^{3}$ Las lesiones cutáneas received December 18, 2017 accepted March 10, 2018 published online May 11, 2018
DOI https://doi.org/ 10.1055/s-0038-1648241. ISSN 0120-789X. eISSN 2027-0119.
Copyright (c) 2019, Sociedad Colombiana License terms de Urología. Publicado por Thieme Revinter Publicações Ltda., Rio de Janeiro, Brazil. Todos los derechos reservados. 
como manifestación de la enfermedad son secundarias a metástasis o incisiones quirúrgicas y la supervivencia media es de aproximadamente veintitrés meses. ${ }^{1}$ El diagnóstico preoperatorio es un desafío y debe sospecharse en pacientes con lesiones paratesticulares o hidrocele con lesiones parietales vegetativas, especialmente si presentan alta vascularización. ${ }^{3}$

\section{Reporte del Caso}

Se trata de un paciente de 71 años de edad, fumador pesado y con antecedente de diabetes mellitus tipo 2, con cuadro clínico de 4 semanas de evolución de aumento del tamaño del contenido escrotal izquierdo. Al examen físico se evidenció hidrocele y una ecografía testicular confirmó la presencia de líquido homogéneo en el saco escrotal izquierdo, con un testículo normal en tamaño y morfología.

El paciente fue llevado a hidrocelectomía mediante incisión transversa en hemiescroto izquierdo. Se encontraron múltiples lesiones vegetativas de entre y 8 milímetros de diámetro involucrando el testículo, el epidídimo y la cara interna de la túnica vaginal (-Fig. 1). Con esos hallazgos, se realizó una biopsia por congelación que reportó la presencia de células malignas. Al no haberse considerado la posibilidad de orquidectomía y no siendo el abordaje escrotal la vía adecuada, se decidió cerrar.

En el posoperatorio, el paciente fue estudiado con PET/CT, el cual no mostró evidencia de metástasis. Una semana más tarde, fue llevado a laparoscopia para descartar diseminación peritoneal y se realizó laparotomía con linfadenectomía retroperitoneal izquierda. El cordón espermático fue liberado a través del canal inguinal y se realizó resección en bloque del hemiescroto y testículo.

Con el informe final de patología se realizó el diagnóstico de mesotelioma epitelial de túnica vaginal, sin invasión tumoral al cordón espermático, escroto o parénquima testicular. Los márgenes de resección fueron negativos. La histología mostraba una neoplasia con patrón papilar y tubulopapilar

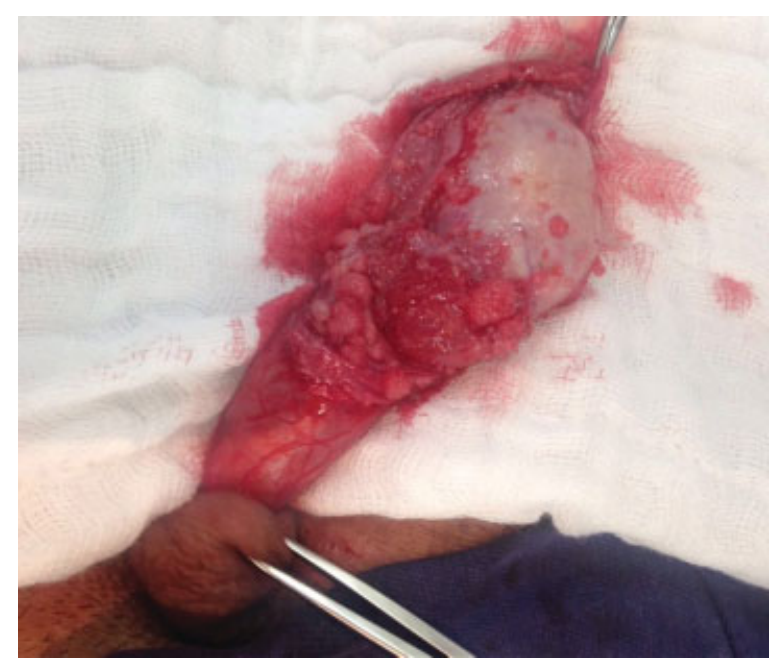

Fig. 1 Múltiples lesiones vegetativas que involucran el testículo, el epidídimo y la cara interna de la túnica vaginal. compuesta por células cuboides y núcleo oval, algunas con pleomorfismo y forma vesicular y otras con nucléolo, con citoplasma escaso a moderado (-Fig. 2A). Se observaron entre siete y diez mitosis. Las estructuras papilares mostraron cambios por hialinización en algunos de los centros fibrovasculares. No había presencia de necrosis. No se evidenció invasión linfovascular ni ganglios positivos, pero se identificó infiltración tumoral al estroma de la túnica vaginal así como al epidídimo (-Fig. 2B). Los estudios de inmunohistoquímica mostraron intensa reactividad de las células tumorales para WT1 (-Fig. 2C), calretinina (-Fig. 2D) y focalmente para D2-40. A un año de seguimiento, el paciente no tiene evidencia de enfermedad.

\section{Discusión}

Los mesoteliomas testiculares se clasificaron inicialmente de acuerdo a la Organización Mundial de la Salud (OMS) como benignos o malignos. ${ }^{4}$ No obstante, en el 2016 se introdujo una nueva clasificación que excluyó el diagnóstico de mesotelioma benigno. ${ }^{5}$ Ese nuevo sistema de clasificación, reconoce el mesotelioma papilar bien diferenciado como una variante con comportamiento indolente. Adicionalmente, el término mesotelioma quístico (antes conocido como mesotelioma benigno), es ahora considerado una entidad no neoplásica.

La evidencia más reciente en referencia al mesotelioma papilar bien diferenciado incluye una revisión de la literatura llevada a cabo por Tan y col. ${ }^{6}$ y publicada en el año 2016. Los autores incluyen ocho reportes de caso de mesotelioma papilar bien diferenciado "verdaderos" basados en criterios histopatológicos estrictos: arquitectura tubulopapilar, baja actividad mitótica, una única fila de células cuboides y ausencia de invasión estromal. Los autores también concluyen que esa entidad puede no estar asociada con la exposición a asbestos, contrario a lo que tradicionalmente se pensaba.

La última clasificación de la OMS no propone cambios para lo que se considera como mesoteliomas malignos. Sin embargo, el sistema de clasificación del 2004 establece que los mesoteliomas paratesticulares pueden dividirse en tres subtipos: epitelial, sarcomatoide o desmoplásico y bifásico, siendo el patrón epitelial el más común, con una frecuencia de hasta el 60\%.,4 Esos tumores pueden originarse a partir de la túnica vaginal o de la túnica albugínea $\mathrm{y}$ representan el segundo tumor paratesticular más frecuente luego de los sarcomas de tejidos blandos. ${ }^{4}$ Cuando se tienen en cuenta todos los mesoteliomas, ellos constituyen solamente el $0,3 \%$ de los casos. ${ }^{7}$ La reactividad para marcadores de inmunohistoquímica como la calretinina y las citoqueratinas $5 / 6$, WT-1, y D2-40 son altamente sugestivos de mesotelioma, especialmente para los subtipos epitelioide y bifásico. ${ }^{4}$

En cuanto a las imágenes diagnósticas, el ultrasonido Doppler ha demostrado utilidad en el diagnóstico inicial de mesoteliomas testiculares, ${ }^{4}$ la tomografía axial computadorizada ha sido utilizada en la estadificación tumoral, y la utilidad del PET/CT ha sido debatida. ${ }^{8}$ Aunque existen pocos reportes en la literatura con respecto a ese último, se considera que es útil en establecer pronóstico, dada su capacidad para identificar lesiones 

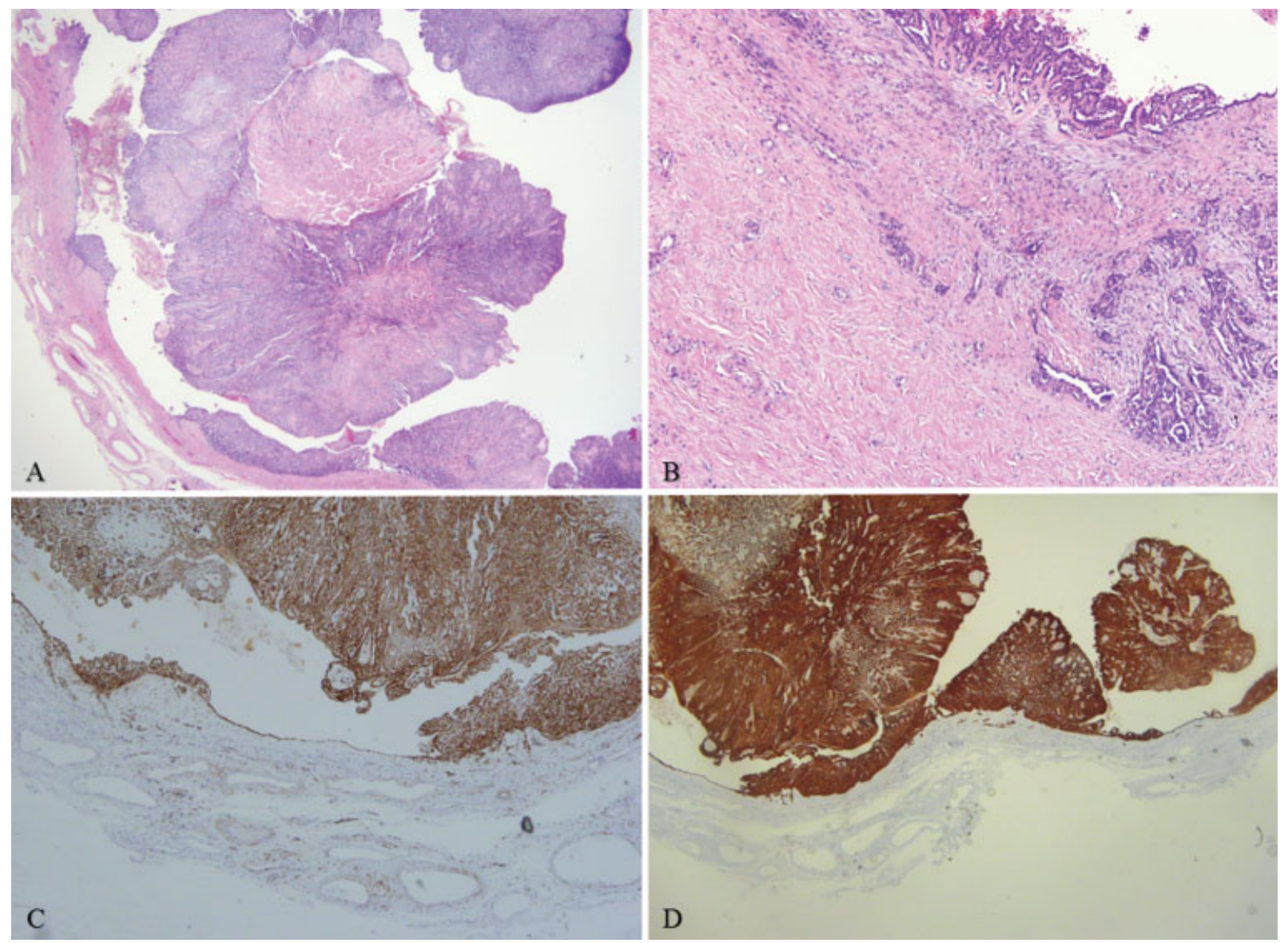

Fig. 2 (A) Tumor dependiente de la túnica vaginal con arquitectura papilar (4X). (B) Infiltración del tumor en el estroma subyacente de la túnica vaginal (20X). (C) Tumor que muestra reactividad intensa para WT1 (10X). (D) Tumor que muestra reactividad intensa para la calretinina (10X).

tumorales en otras localizaciones. Esas lesiones se caracterizan por ser depósitos hipermetabólicos y representan invasión directa o diseminación. ${ }^{8}$ En nuestro caso, no se encontró evidencia de metástasis, por lo que no se propuso manejo adicional a la cirugía.

El manejo multimodal del mesotelioma testicular incluye orquidectomía con resección en bloque de la túnica vaginal y el escroto. La linfadenectomía retroperitoneal tiene fines diagnósticos y terapéuticos. ${ }^{9}$ Por otro lado, la radioterapia puede incluirse como estrategia preventiva, como parte del manejo paliativo o como tratamiento de recaída local. ${ }^{2} \mathrm{La}$ quimioterapia se ha empleado en enfermedad metastásica, aunque su impacto es bastante limitado. ${ }^{10}$

Este es el segundo caso de mesotelioma de túnica vaginal reportado en la literatura colombiana, siendo el primero el presentado por Ramírez y cols. ${ }^{11}$ en el XXXVI Congreso de la Sociedad Colombiana de Urología en el año 2001, quienes describieron un cuadro clínico similar y una histología de tipo bifásico.

\section{Conclusiones}

El mesotelioma de la túnica vaginal es una entidad rara e infrecuente que se debe sospechar en pacientes con hidrocele de rápida progresión y/o ante la presencia de lesiones hipervascularizadas y pediculadas en el ultrasonido Doppler. Se requiere un diagnóstico oportuno a fin de mejorar el pronóstico y la supervivencia de esos pacientes. La orquidectomía radical más la linfadenectomía retroperitoneal, es el estándar de manejo.

\section{Agradecimientos}

Este reporte de caso no recibió ayuda externa.

\section{Conflictos de Interés}

Ninguno.

\section{Financiación}

No se recibió ningún tipo de financiación.

\section{Consideraciones Éticas}

El paciente firmó un consentimiento informado para permitir la publicación de este reporte de caso. El presente reporte de caso fue aprobado previamente por el Comité de Ética en Investigación de nuestra institución.

\section{Bibliografía}

1 Hispán P, Pascual JC, González I, Bravo D, Peiró G. Cutaneous Metastases From Malignant Mesothelioma of the Tunica Vaginalis Testis. Am J Dermatopathol 2016;38(03):222-225 
2 Erdogan S, Acikalin A, Zeren H, Gonlusen G, Zorludemir S, Izol V. Well-differentiated papillary mesothelioma of the tunica vaginalis: a case study and review of the literature. Korean J Pathol 2014;48(03):225-228

3 Bertolotto M, Boulay-Coletta I, Butini R, et al. Imaging of mesothelioma of tunica vaginalis testis. Eur Radiol 2016;26 (03):631-638

4 Eble JN, Sauter G, Epstein JI, Sesterhenn IA. Pathology and Genetics of Tumours of the Urinary System and Male Genital Organs. 2004

5 Moch H, Cubilla AL, Humphrey PA, Reuter VE, Ulbright TM. The 2016 WHO Classification of Tumours of the Urinary System and Male Genital Organs-Part A: Renal, Penile, and Testicular Tumours. Eur Urol 2016;70(01):93-105

6 Tan WK, Tan M-Y, Tan WS, et al. Well-Differentiated Papillary Mesothelioma of the Tunica Vaginalis: Case Report and
Systematic Review of Literature. Clin Genitourin Cancer 2016;14 (04):e435-e439http://linkinghub.elsevier.com/retrieve/pii/S1558 767316300611 [Internet]

7 Murai Y. Malignant mesothelioma in Japan: analysis of registered autopsy cases. Arch Environ Health 2001;56(01):84-88

8 Ozguven S, Aras M, Dede F, Ones T, Turoglu HT. Scrotal peritoneal mesothelioma on PET/CT. Clin Nucl Med 2014;39(12):1045-1046

9 Haldipur N, Devaraj S, Shehata A, et al. Retroperitoneal lymph node dissection for metastatic germ cell tumours. Ann R Coll Surg Engl 2011;93(04):301-305

10 Hai B, Yang Y, Xiao Y, Li B, Chen C. Diagnosis and prognosis of malignant mesothelioma of the tunica vaginalis testis. Can Urol Assoc J 2012;6(06):E238-E241

11 Ramirez E, Varela R, Mosquera Angulo JE. Mesotelioma Maligno de la Túnica Vaginal: Reporte de un caso y Revisión de la Literatura (Poster). Urol Colomb 2001; Suppl 02 